\title{
Combinatorics of Positroids
}

\author{
Suho $\mathrm{Oh}^{1 \rrbracket}$
}

Department of Mathematics, Massachusetts Institute of Technology, 77 Massachusetts Ave, Cambridge, MA 02139

Recently Postnikov gave a combinatorial description of the cells in a totally-nonnegative Grassmannian. These cells correspond to a special class of matroids called positroids. There are many interesting combinatorial objects associated to a positroid. We introduce some recent results, including the generalization and proof of the purity conjecture by Leclerc and Zelevinsky on weakly separated sets.

Keywords: Positroids, Total positivity, Grassmannian, Matroids, $\lrcorner$-diagrams, Decorated permutations, Weakly separated.

\section{Introduction}

A positroid is a matroid that can be represented by a $k \times n$ matrix with nonnegative maximal minors. The classical theory of total positivity concerns matrices in which all minors are non-negative, and this subject was extended by Lusztig.

Lusztig introduced the totally non-negative variety $G \geq 0$ in an arbitrary reductive group $G$ and the totally non-negative part $(G / P)_{\geq 0}$ of a real flag variety $(G / P)$. He also conjectured that $(G / P)_{\geq 0}$ is made up of cells, and this was proved by Rietsch.

In this paper, we will restrict our attention to $\left(G r_{k n}\right)_{\geq 0}$, the totally non-negative Grassmannian. Then there is a more refined decomposition using matroid strata. Recently, Postnikov obtained a relationship between $\left(G r_{k n}\right)_{\geq 0}$ and certain planar bicolored graphs, producing a combinatorially explicit cell decomposition of $\left(G r_{k n}\right) \geq 0$. The cells correspond to positroids.

In this extended abstract, We will briefly sketch some results about positroids, and introduce some open problems. No proof will be provided.

In section 3, we will go over describing matroidal operations on positroids via decorated permutations. In section 4, we will describe positroids by forbidden minors. In section 5 and 6, we will introduce the proof of the purity conjecture given in ( $\mathrm{OPS})$, and introduce related enumerative problems.

\section{Preliminaries}

An element in the Grassmannian $G r_{k n}$ can be understood as a collection of $n$ vectors $v_{1}, \cdots, v_{n} \in \mathbb{R}^{k}$ spanning the space $\mathbb{R}^{k}$ modulo the simultaneous action of $G L_{k}$ on the vectors. The vectors $v_{i}$ are the

\footnotetext{
${ }^{\dagger}$ Supported in part by Samsung Scholarship.

1365-8050 @ 2009 Discrete Mathematics and Theoretical Computer Science (DMTCS), Nancy, France
} 
columns of a $k \times n$-matrix $A$ that represents the element of the Grassmannian. Then an element $V \in G r_{k n}$ represented by $A$ gives the matroid $\mathcal{M}_{V}$ whose bases are the $k$-subsets $I \subset[n]$ such that $\Delta_{I}(A) \neq 0$. Here, $\Delta_{I}(A)$ denotes the determinant of $A_{I}$, the $k$ by $k$ submatrix of $A$ in the column set $I$.

Then $G r_{k n}$ has a subdivision into matroid strata $S_{\mathcal{M}}$ labelled by some matroids $\mathcal{M}$ :

$$
S_{\mathcal{M}}:=\left\{V \in G r_{k n} \mid \mathcal{M}_{V}=\mathcal{M}\right\}
$$

The elements of the stratum $S_{\mathcal{M}}$ are represented by matrices $A$ such that $\Delta_{I}(A) \neq 0$ if and only if $I \in \mathcal{M}$. Now we define the Schubert matroids, which correspond to the cells of the matroid strata.

Ordering $<_{w}, w \in S_{n}$ is defined as $a<_{w} b$ if $w^{-1} a<w^{-1} b$ for $a, b \in[n]$.

Definition 1 Let $A, B \in\left(\begin{array}{c}{[n]} \\ k\end{array}\right), w \in S_{n}$ where

$$
\begin{aligned}
& A=\left\{i_{1}, \cdots, i_{k}\right\}, i_{1}<_{w} i_{2}<_{w} \cdots<_{w} i_{k} \\
& B=\left\{j_{1}, \cdots, j_{k}\right\}, j_{1}<_{w} j_{2}<_{w} \cdots<_{w} j_{k}
\end{aligned}
$$

Then we set $A \leq_{w} B$ if and only if $i_{1} \leq_{w} j_{1}, \cdots, i_{k} \leq_{w} j_{k}$. This ordering is called the Gale ordering on $\left(\begin{array}{c}{[n]} \\ k\end{array}\right)$ induced by $w$. We denote $\leq_{t}$ for $t \in[n]$ as $<_{c^{t-1}}$ where $c=(1, \cdots, n) \in S_{n}$.

We can also define matroids from the above ordering. See $(\bar{G}),(\overline{B G W})$.

Definition 2 Let $\mathcal{M} \subseteq\left(\begin{array}{c}{[n]} \\ k\end{array}\right)$. Then $\mathcal{M}$ is a matroid if and only if $\mathcal{M}$ satisfies the following property. For every $w \in S_{n}$, the collection $\mathcal{M}$ contains a unique member $A \in \mathcal{M}$ maximal in $\mathcal{M}$ with respect to the partial order $\leq_{w}$.

Now we can define a Schubert matroid and a dual Schubert matroid using the partial order $\leq_{w}$.

Definition 3 For $I=\left(i_{1}, \cdots, i_{k}\right)$, the Schubert Matroid $S M_{I}^{w}$ consists of bases $H=\left(j_{1}, \cdots, j_{k}\right)$ such that $I \leq_{w} H$. The dual Schubert matroid $\tilde{S M}_{I}^{w}$ consists of bases $H=\left(j_{1}, \cdots, j_{k}\right)$ such that $I \geq_{w} H$.

Let us define the totally nonnegative Grassmannian and its cells.

Definition 4 ((宇) The totally nonnegative Grassmannian $G r_{k n}^{t n n} \subset G r_{k n}$ is the quotient $G r_{k n}^{t n n}=G L_{k}^{+} \backslash M a t_{k n}^{t n n}$, where $M a t_{k n}^{t n n}$ is the set of real $k \times n$-matrices $A$ of rank $k$ with nonnegative maximal minors $\Delta_{I}(A) \geq 0$ and $G L_{k}^{+}$is the group of $k \times k$-matrices with positive determinant.

Definition $5(\underline{(\mathbf{P})})$ Totally nonnegative Grassmann cells $S_{\mathcal{M}}^{t n n}$ in $G r_{k n}^{t n n}$ are defined as $S_{\mathcal{M}}^{t n n}:=S_{\mathcal{M}} \cap$ $G r_{k n}^{t n n} . \mathcal{M}$ is called a positroid if the cell $S_{\mathcal{M}}^{t n n}$ is nonempty.

Note that from above definitions, we get

$$
S_{\mathcal{M}}^{t n n}=\left\{G L_{k}^{+} \bullet A \in G r_{k n}^{t n n} \mid \Delta_{I}(A)>0 \text { for } I \in \mathcal{M}, \Delta_{I}(A)=0 \text { for } I \notin \mathcal{M}\right\} .
$$

In $(\mathrm{P})$, Postnikov showed a bijection between each cell and a combinatorial object called Grassmann necklace. He also showed that those necklaces can be represented as objects called decorated permutations. Let's first see how they are defined.

Definition $6((\mathbf{P}))$ A Grassmann necklace is a sequence $\mathcal{I}=\left(I_{1}, \cdots, I_{n}\right)$ of subsets $I_{r} \subseteq[n]$ such that, for $i \in[n]$, if $i \in I_{i}$ then $I_{i+1}=\left(I_{i} \backslash\{i\}\right) \cup\{j\}$, for some $j \in[n]$; and if $i \in I_{i}$ then $I_{i+1}=I_{i}$. (Here the indices are taken modulo $n$.) In particular, we have $\left|I_{1}\right|=\cdots=\left|I_{n}\right|$. 
Definition $7((\mathbf{P}))$ A decorated permutation $\pi^{:}=(\pi, c o l)$ is a permutation $\pi \in S_{n}$ together with a coloring function col from the set of fixed points $\{i \mid \pi(i)=i\}$ to $\{1,-1\}$. That is, a decorated permutation is a permutation with fixed points colored in two colors.

It is easy to see the bijection between necklaces and decorated permutations. To go from a Grassmann necklace $\mathcal{I}$ to a decorated permutation $\pi^{:}=(\pi, \mathrm{col})$.

- if $I_{i+1}=\left(I_{i} \backslash\{i\}\right) \cup\{j\}, j \neq i$, then $\pi(i)=j$

- if $I_{i+1}=I_{i}$ and $i \notin I_{i}$ then $\pi(i)=i, \operatorname{col}(i)=1$

- if $I_{i+1}=I_{i}$ and $i \in I_{i}$ then $\pi(i)=i, \operatorname{col}(i)=-1$.

To go from a decorated permutation $\pi^{:}=(\pi, \mathrm{col})$ to a Grassmann necklace $\mathcal{I}$,

$$
I_{r}=\left\{i \in[n] \mid i<_{r} \pi^{-1}(i) \text { or }(\pi(i)=i \text { and } \operatorname{col}(i)=-1)\right\} .
$$

Let's look at a simple example. For decorated permutation $\pi^{:}$with $\pi=81425736$ and $\operatorname{col}(5)=1$, we get $I_{1}=\{1,2,3,6\}, I_{2}=\{2,3,6,8\}, I_{3}=\{3,6,8,1\}, I_{4}=\{4,6,8,1\}, I_{5}=\{6,8,1,2\}, I_{6}=$ $\{6,8,1,2\}, I_{7}=\{7,8,1,2\}, I_{8}=\{8,1,2,3\}$.

Recall that we have defined $<_{r}$ to be a total order on $[n]$ such that $r<_{r} r+1<_{r} \cdots<_{r} n<_{r} 1<_{r}$ $\cdots<_{r} r-1$. This ordering is the same as $<_{c^{r-1}}$ where $c=(1, \cdots, n) \in S_{n}$.

Lemma $8((\overline{\mathbf{P}}))$ For a matroid $\mathcal{M} \subseteq\left(\begin{array}{c}{[n]} \\ k\end{array}\right)$ of rank $k$ on the set $[n]$, let $\mathcal{I}_{\mathcal{M}}=\left(I_{1}, \cdots, I_{n}\right)$ be the sequence of subsets such that $I_{i}$ is the minimal member of $\mathcal{M}$ with respect to $\leq_{i}$. Then $\mathcal{I}_{\mathcal{M}}$ is a Grassmann necklace.

Theorem $9(\overline{\mathbf{P}})$ Let $S_{\mathcal{M}}^{t n n}$ be a nonnegative Grassmann cell, and let $\mathcal{I}_{\mathcal{M}}=\left(I_{1}, \cdots, I_{n}\right)$ be the Grassmann necklace corresponding to $\mathcal{M}$. Then

$$
S_{\mathcal{M}}^{t n n}=\bigcap_{i=1}^{n} \Omega_{I_{i}}^{c^{i-1}} \cap G r_{k n}^{t n n}
$$

where $c=(1, \cdots, n) \in S_{n}$ and $\Omega_{I_{i}}^{c^{i-1}}$ is the permuted Schubert cell, which is the set of elements $V \in$ $G r_{k n}$ such that $I_{i}$ is the lexicographically minimal base of $M_{V}$ with respect to ordering $<_{w}$ on $[n]$.

This theorem implies that bases of a positroid are included in each Schubert matroids corresponding to the Grassmann necklace, but it does not imply that they are equal. Postnikov therefore conjectured that each positroid is exactly the intersection of Schubert matroids.

Theorem 10 ((区1) $\mathcal{M}$ is a positroid if and only if for some Grassmann necklace $\left(I_{1}, \cdots, I_{n}\right)$,

$$
\mathcal{M}=\bigcap_{i=1}^{n} S M_{I_{i}}^{c^{i-1}} .
$$

In other words, $\mathcal{M}$ is a positroid if and only if the following holds : $H \in \mathcal{M}$ if and only if $H \geq_{t} I_{t}$ for any $t \in[n]$.

Let's see an example. Let $\mathcal{M}$ be a positroid indexed by a decorated permutation $[5,3,2,1,4,6]$, with $\operatorname{col}(6)=-1$. Then we get : $I_{1}=\{1,2,4,6\}, I_{2}=\{2,4,5,6\}, I_{3}=\{3,4,5,6\}, I_{4}=\{4,5,6,2\}, I_{5}=$ $\{5,6,1,2\}, I_{6}=\{1,2,4,6\}$.

$$
\mathcal{M}=\{\{1,2,4,6\},\{1,2,5,6\},\{1,3,4,6\},\{1,3,5,6\},\{2,4,5,6\},\{3,4,5,6\}\} .
$$




\section{Matroid operations via decorated permutations}

In this section we will see that matroidal contraction, deletion and dual for positroids can be described via operations on decorated permutations. In this section, we will use - for the set subtraction.

Definition 11 Given a matroid $\mathcal{M}$ on set $E$. The contraction of $T \subset E$ from $\mathcal{M}$ is defined as

$$
\mathcal{M} / T=\{I-T: T \subset I \in \mathcal{M}\}
$$

The deletion of $T \subset E$ from $\mathcal{M}$ is defined as

$$
\mathcal{M} \backslash T=\{I \in \mathcal{M}: I \subset(E-T)\} .
$$

The restriction of $\mathcal{M}$ to $T \subset E$ is defined as

$$
\left.\mathcal{M}\right|_{T}=\mathcal{M} \backslash(E-T)
$$

Fix a decorated permutation $\pi^{:}=(\pi, \mathrm{col})$. Let $\left(I_{1}, \cdots, I_{n}\right)$ be the corresponding Grassmannian necklace and $\mathcal{M}$ the corresponding positroid. Now look at $\left(J_{1}:=\pi^{-1}\left(I_{1}\right), \cdots, J_{n}:=\pi^{-1}\left(I_{n}\right)\right)$. They also form a necklace. We will call this the upper Grassmann necklace of $\pi$. So we have the following theorem.

Theorem 12 ((О1)) Pick a decorated permutation. $\pi$ : Then we have the corresponding Grassmann necklace and upper Grassmann necklace $\mathcal{I}=\left(I_{1}, \cdots, I_{n}\right), \mathcal{J}=\left(J_{1}, \cdots, J_{n}\right)$. Then $J_{i}=\pi^{-1}\left(I_{i}\right)$ for all $i \in[n]$ where $\pi^{:}=(\pi, \mathrm{col})$. And we have the equality

$$
\bigcap_{i=1}^{n} S M_{I_{i}}^{c^{i-1}}=\bigcap_{i=1}^{n} S M_{J_{i}}^{c^{i-1}}
$$

As a corollary, we now know how to describe taking the dual of positroids in terms of decorated permutations.

Corollary 13 Let $\mathcal{M}$ be a positroid indexed by $\pi^{:}=(\pi$, col $)$. Let $\mathcal{M}^{\prime}$ be a matroid obtained by taking the dual of $\mathcal{M}$. Then $\mathcal{M}^{\prime}$ is a positroid and $\mathcal{M}^{\prime}$ is indexed by $\left(\pi^{-1},-c o l\right)$.

Now let's show how to describe contractions of positroids via decorated permutations. Let $\mathcal{M}$ be a positroid indexed by $\pi^{:}$. Denote $\mathcal{M}^{\prime}$ be $\mathcal{M} /\{j\}, j \in[n]$. Let $\mu^{:}$be the decorated permutation indexing $\mathcal{M}^{\prime}$. We have an algorithm to obtain $\mu^{:}$directly from $\pi^{:}$.

For $i \neq j$, if $\operatorname{col}(i)$ is defined, $\operatorname{col}^{\prime}(i)$ is defined as the same value. For other $i \in[n]$, if $\mu(i)=i$, $\operatorname{col}^{\prime}(i)=+1$. Let's try out the algorithm for $\pi=[6,1,4,8,2,7,3,5]$ and $j=3$.

1. $\mu=[6,1,4,8,2,7,3,5]$.

2. $\mu(3)=3, q=4$. Now $a=4, \mu=[6,1,3,8,2,7,3,5]$.

3. Since $q=a=4, \mu(4)=4, q=\pi(4)=8$. Now $a=5, \mu=[6,1,3,4,2,7,3,5]$.

4. Since $q \neq a, \pi(5)=2<_{6} 3, \mu(5)=\min _{6}(2,8)=8, q=\max _{6}(2,8)=2$. Now $a=6, \mu=$ $[6,1,3,4,8,7,3,5]$. 


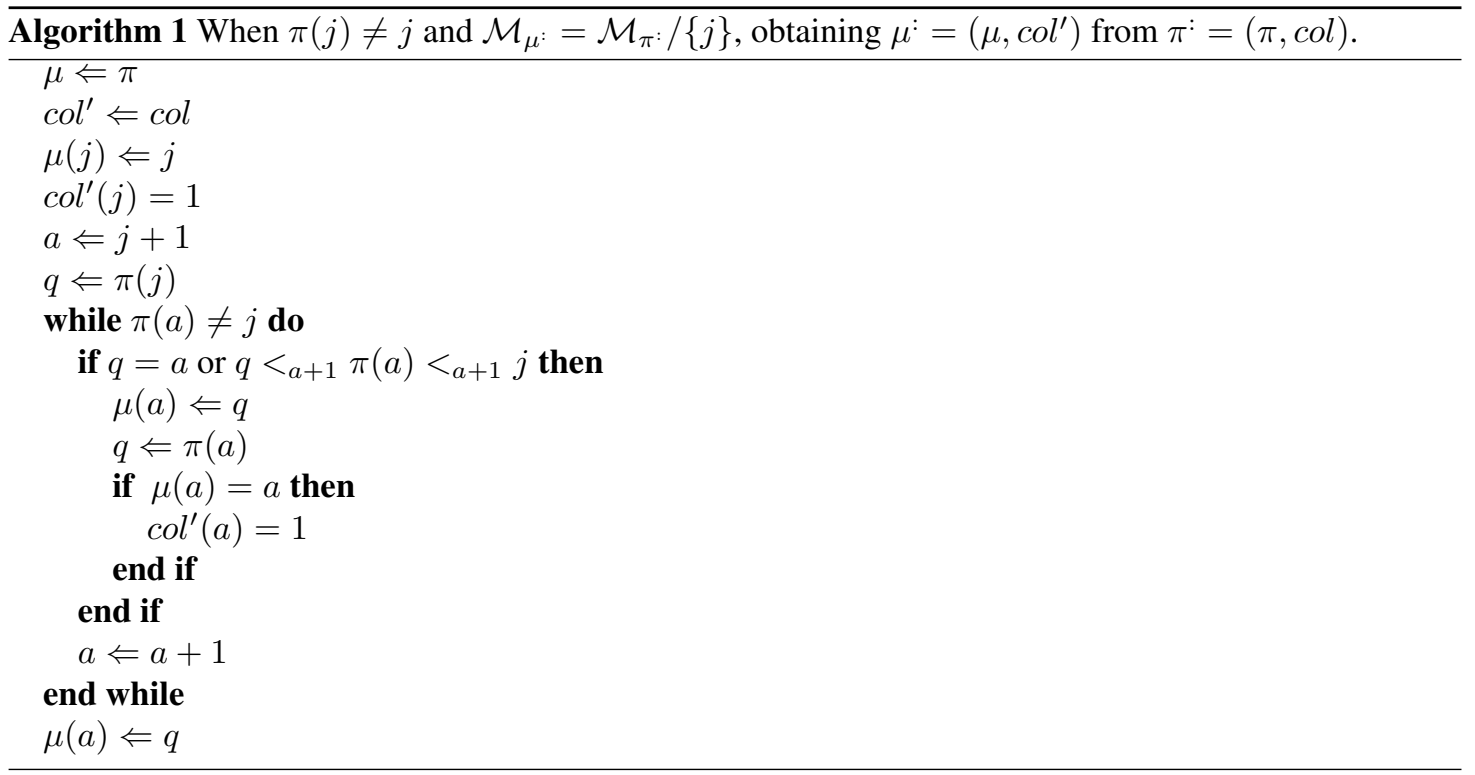

5. Since $q \neq a, \pi(6)=7<<_{7} 3, \mu(6)=\min _{7}(2,7)=7, q=\max _{7}(2,7)=2$. Now $a=7, \mu=$ $[6,1,3,4,8,7,3,5]$.

6. Since $\pi(7)=3, \mu(7)=2$. We are finished and the result is $\mu=[6,1,3,4,8,7,2,5]$.

7. $\operatorname{col}^{\prime}(3)=+1, \operatorname{col}^{\prime}(4)=+1$.

The deletion of $\mathcal{M}$ by $\{j\}$ can also be obtained directly by an algorithm. See (02). It will be interesting to check how the contraction and restriction of positroids can be described in terms of $\lrcorner$-diagrams, which we will show the definition in the next section.

Problem 14 How can contraction and restriction of positroids described in terms of $\lrcorner$-diagrams?

\section{Positroids and Forbidden Minors}

Another important property of positroids is that they can be characterized via forbidden minors. A matroid is called a minor of $\mathcal{M}$ if it can be obtained by sequence of restrictions and contractions from $\mathcal{M}$.

Lemma 15 Let $\mathcal{M}$ be a matroid of rank $k$ over $[n] . \mathcal{M}$ is a positroid if and only if it satisfies the following condition:

Let $T$ be any $k-2$ element subset of $[n]$. For each $a, b, c, d \in[n]-T$ be such that $a<_{t} b<_{t} c<_{t} d$ for some $t \in[n]$, the following relation holds. $T \cup\{a, c\}, T \cup\{b, d\} \in \mathcal{M}$ if and only $T \cup\{a, b\}, T \cup\{c, d\} \in$ $\mathcal{M}$ or $T \cup\{a, d\}, T \cup\{b, c\} \in \mathcal{M}$. See Figure 1$]$

Notice that the above condition can also be written as the following. Let $T$ be any $k-2$ element subset of $[n]$. For any 4 element subset $Q \subseteq[n]-T,\left.(\mathcal{M} / T)\right|_{Q}$ is a positroid. 


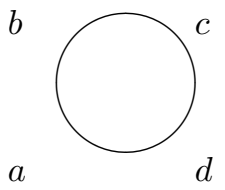

Fig. 1: $a<_{t} b<_{t} c<_{t} d$

Let's find all the matroids of rank 2 over [4] that are not positroids:

$$
\{12,34,13,23,14\},\{12,34,14,23,24\},\{12,34,14,23\} .
$$

By Lemma 15 and remark following it, we can conclude as the following.

Theorem $16(\underline{(\mathbf{O 1})})$ A matroid is a positroid if and only if it has no minors among the above list.

We can look at the nonnegative Grassmannian over finite fields. The positive part of a $\mathbb{F}_{q}$ when $q$ is prime is defined as set of elements that can be expressed as a square of a nonzero element inside that field. Denote this as $\mathbb{F}_{q}^{+}$. So we can define cells in the nonnegative Grassmannian of $\mathbb{F}_{q}$. Using similar notation as before,

$$
S_{\mathcal{M}}^{t n n}\left(\mathbb{F}_{q}\right):=\left\{G L_{k}^{+}\left(\mathbb{F}_{q}\right) \bullet A \in G r_{k n}\left(\mathbb{F}_{q}\right) \mid \Delta_{I}(A) \in \mathbb{F}_{q}^{+} \text {for } I \in \mathcal{M}, \Delta_{I}(A)=0 \text { for } I \notin \mathcal{M}\right\} .
$$

$\mathbb{F}_{q}$-positroid is defined as matroid $\mathcal{M}$ such that $S_{\mathcal{M}}^{\operatorname{tnn}}\left(\mathbb{F}_{q}\right)$ is nonempty. The Plücker relations behave nicely in $\mathbb{F}_{3}$ and $\mathbb{F}_{7} . \mathbb{F}_{3}, \mathbb{F}_{7}$ positroids are special cases of positroids, and can also be expressed in terms of forbidden minors.

Proposition 17 A positroid is a $\mathbb{F}_{3}, \mathbb{F}_{7}$-positroid if and only if it avoids minor $\{12,34,13,23,14,24\}$.

\section{Plabic graphs and $\lrcorner$-diagrams.}

In $(\mathbb{P})$, Postnikov defined plabic graphs and $\lrcorner$-diagrams. There are a number of plabic graphs that can be used to paramatrize the corresponding positroid strata. And for each positroid strata, there exists a unique plabic graph that corresponds to a combinatorial object called the $\lrcorner$-diagram. So there is a bijection between positroids and $\lrcorner$-diagrams. In ( $(\overline{\mathrm{OPS}})$, we used plabic graphs to describe collection of maximal weakly separated sets of Leclerc and Zelevinsky. And in the next section, we will use $\lrcorner$-diagrams to count a related invariant on weakly separated sets. For more details on plabic graphs and $\lrcorner$-diagrams, see $(\mathbb{P})$.

Definition 18 A planar bicolored graph, or simply a plabic graph is a planar undirected graph $G$ drawn inside a disk. The vertices on the boundary are called boundary vertices. All vertices in the graph are colored either white or black.

A plabic graph is associated with a decorated permutation. The positroid corresponding to the decorated permutation is exactly the positroid that the plabic graph gives a parametrization of the corresponding strata. 
Definition 19 For a plabic graph $G$, a trip (one-way trip in notion of $(\mathbb{P})$ ) is a directed path $T$ in $G$ such that $T$ joins two boundary vertices and satisfies the following rules of the road. Turn right at a black vertex, and turn left at a white vertex. Trip permutation $\pi_{G} \in S_{n}$ is defined such that $\pi_{G}(i)=j$ whenever trip that starts at the boundary vertex labeled $i$ ends at boundary vertex $j$. Decorated trip permutation $\pi_{G}^{:}$is defined similarly.

We have following 3 moves on plabic graphs.

(M1) Pick a square with vertices alternating in colors. Then we can switch the colors of all the vertices. See Figure 2
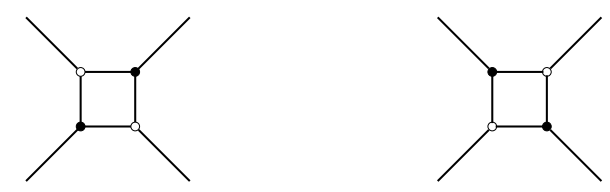

Fig. 2: (M1) Square move

(M2) For two adjoint vertices of the same color, we can contract them into one vertice. See Figure 3
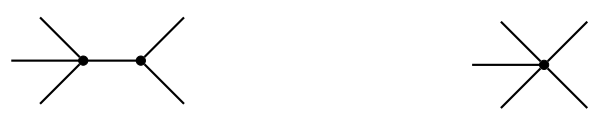

Fig. 3: (M2) Unicolored edge contraction

(M3) We can insert or remove a vertice inside any edge. See Figure 4

Fig. 4: (M3) Middle vertex insertion/removal

Now we define reducedness of plabic graphs.

Definition 20 A strand $i \in[n]$ for a plabic graph is given by the trip starting at $\pi^{-1}(i)$ and ending at $i$. Then the plabic graph is reduced if it satisfies the following two properties:

1. Each strand does not self-intersect.

2. For any two strands that intersect, the direction they are heading should be opposite of each other. That is, if they intersect at points $a$ and $b$, the one should be heading from a to $b$ and another from $b$ to $a$.

Really nice about the property of being a plabic graph is that it is closed under certain combinatorial moves. The same goes for being a reduced plabic graph.

Theorem $21\left((\mathbf{P})\right.$ Let $G$ and $G^{\prime}$ be two (reduced) plabic graphs with the same number of boundary vertices. Then the following claims are equivalent: 
- $G$ can be obtained from $G^{\prime}$ by moves (M1)-(M3).

- These two graphs have the same decorated trip permutation $\pi_{G}^{:}=\pi_{G^{\prime}}^{:}$.

We now define what is called a J-diagram, which encodes one unique reduced plabic graph of the corresponding positroid. They are nice combinatorial objects that can be used to easily compute various invariants of the corresponding positroid. One example is that the dimension of the strata equals the number of dots plus one. Another example will be introduced in the next section.

Definition 22 A Young diagram of shape $\lambda$ is called a $\lrcorner$-diagram if it satisfies the following property. Each box is either empty or filled with one dot. For any three boxes indexed $(i, j),\left(i^{\prime}, j\right),\left(i, j^{\prime}\right)$, where $i^{\prime}<i$ and $j^{\prime}<j$, if boxes on position $\left(i^{\prime}, j\right)$ and $\left(i, j^{\prime}\right)$ contain a dot inside, then the box on $(i, j)$ also contains a dot. This property is called the $\lrcorner$-property.
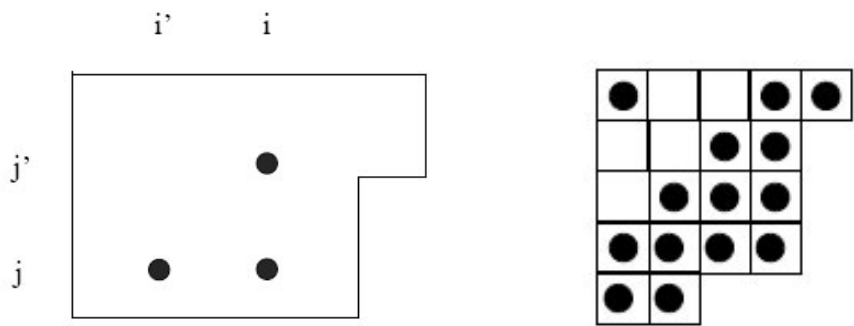

Fig. 5: $\lrcorner$-property and an example of a $\lrcorner$-diagram

The boundary of the diagram forms a lattice path from the upper-right corner to lower-left corner. Label the $n$ steps in this path by numbers $1, \cdots, n$ consecutively. Define $I(\lambda)$ as the set of lables of $k$ vertical steps in the path. Put dots on each edge of the boundary path. Connect all dots on same row and connect all dots on the same column. Then we get a $\lrcorner$-graph.

Definition 23$\lrcorner$-graph is obtained from a $\lrcorner$-diagram in the following way. Put a dot on center of each edge of the boxes on the southeast boundary of the diagram, and label them $1,2, \cdots$ starting from northeast to southwest. Call these the boundary vertices. Now for each dot inside the $\lrcorner$-diagram, draw a horizontal line to its right, and vertical line to its bottom until it reaches the boundary of the diagram.

Now below is the method to check which decorated permuation the $\lrcorner$-diagram corresponds to. That is, it tells us which positroid the 」-diagram corresponds to.

Theorem $24((\mathbb{P}))$ Define a map $\chi$ that sends a $\lrcorner$-diagram to a decorated permutation $\pi^{:}=(\pi, \mathrm{col})$ defined as following. Set $\pi(i)=j$ where we reach $j$ when we start from $i$ and follow the rules of the road in Figure 7 If $\pi(i)=i$, set col $(i)=-1$ if $i$ is on a horizontal edge, $\operatorname{col}(i)=1$ if otherwise. Then $\chi$ is a bijection between $\rfloor$-diagrams having lower boundary $I$ and decorated permutations having $I_{1}=I$, where $\mathcal{I}=\left(I_{1}, \cdots, I_{n}\right)$ is the Grassmann necklace of the decorated permutation. 

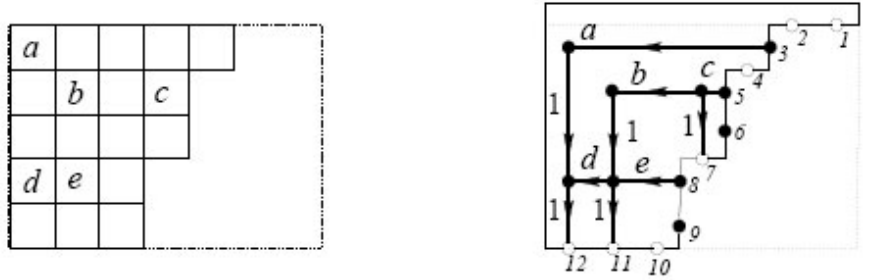

Fig. 6: Example of a $\lrcorner$-graph
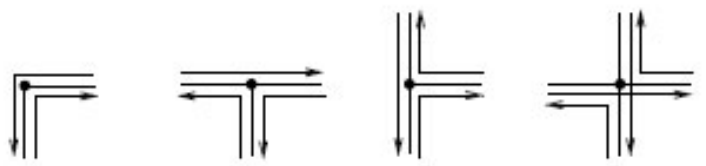

Fig. 7: Rules of the road

\section{Weak Separation and the conjectures of Leclerc and Zelevin- sky}

In this section, we introduce Leclerc and Zelevinsky's conjecture on weakly separated sets, which was recently generalized and proved in (OPS) using positroids and plabic graphs. And then, we show a nice invariant related to this problem, counted using $\lrcorner$-diagrams.

We say that $\alpha_{1}, \alpha_{2}, \cdots, \alpha_{n}$ are circularly ordered if there exists $\alpha \in[n]$ such that $\alpha_{1}<_{\alpha} \cdots<_{\alpha} \alpha_{n}$.

Definition 25 ((LZ)) Let $I, J \in\left(\begin{array}{c}{[n]} \\ k\end{array}\right)$. We say that $I$ and $J$ are weakly separated if there does not exist $i_{1}, i_{2} \in I \backslash J, j_{1}, j_{2} \in J \backslash I$ such that $i_{1}, j_{1}, i_{2}, j_{2}$ are circularly ordered. For $\mathcal{C} \subset\left(\begin{array}{c}{[n]} \\ k\end{array}\right)$, we call $\mathcal{C}$ a maximal weakly separated collection if it is maximal among collections such that each of its elements are pairwise weakly separated.

Leclerc and Zelevinsky observed the following:

Proposition $26(\overline{(\mathbf{L Z})})$ Let $S \in\left(\begin{array}{c}{[n]} \\ k-2\end{array}\right)$ and let $\alpha, \beta, \gamma, \delta \in[n] \backslash S$ be circularly ordered. Suppose that a maximal weakly separated collection $\mathcal{C}$ contains $S \alpha \beta, S \beta \gamma, S \gamma \delta, S \delta \alpha$ and $S \alpha \gamma$. Then $\mathcal{C}^{\prime}:=\mathcal{C} \backslash\{S \alpha \gamma\} \cup$ $\{S \beta \delta\}$ is also a maximal weakly separated collection.

In the above proposition, we say that $\mathcal{C}^{\prime}$ and $\mathcal{C}$ are obtained from each other by a flip. If $\mathcal{C}^{\prime}$ can be obtained from $\mathcal{C}$ by sequence of flips, we say that $\mathcal{C}^{\prime}$ and $\mathcal{C}$ are flip-connected.

Leclerc and Zelevinsky formulated two challenging conjectures on maximal weakly separated collections and they were recently proved in ( (OPS). The main tool is to relate maximal weakly separated collection and plabic graphs. There is a nice way to label the faces of plabic graphs corresponding to positroid $\mathcal{M} \subseteq\left(\begin{array}{c}{[n]} \\ k\end{array}\right)$ with elements of $\left(\begin{array}{c}{[n]} \\ k\end{array}\right)$. Since each strand divides a disk into two parts, each face is either on the left side or the right side of a strand. For each face we label that face with $J \in\left(\begin{array}{c}{[n]} \\ k\end{array}\right)$ such 


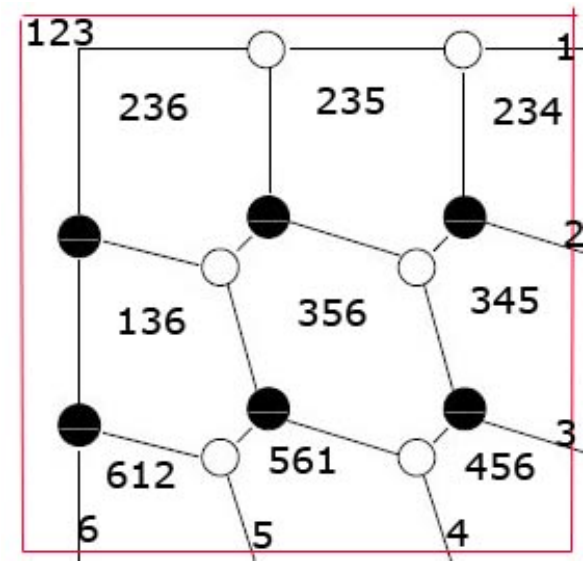

Fig. 8: Labeling faces of a plabic graph

that $i \in J$ if and only if there is a strand containing that face on its left side. See figure 8 for an example. So given a plabic graph $\mathcal{G}$, we define $\mathcal{F}_{\mathcal{G}}$ as the set of labels that occur on each face of that graph.

Theorem 27 (鸟S) $) \mathcal{C} \subseteq\left(\begin{array}{c}{[n]} \\ k\end{array}\right)$ is a maximal weakly separated collection if and only if it is $\mathcal{F}_{\mathcal{G}}$ for some $\mathcal{G}$ a reduced plabic graph of positroid $\mathcal{M}=\left(\begin{array}{c}{[n]} \\ k\end{array}\right)$.

For example, $\{123,234,345,456,561,612,236,235,136,356\}$ is a maximal weakly separated collection of $\left(\begin{array}{c}{[6]} \\ 3\end{array}\right)$. And one can check from Figure 8 that this collection comes from labeling the faces of a plabic graph of the positroid $\left(\begin{array}{c}{[6]} \\ 3\end{array}\right)$.

In $(\mathbb{P})$, it shown that the number of faces of reduced plabic graphs of the same positroid are the same, and that they equal the dimension of the corresponding strata. So as a corollary of the theorem, the two conjectures of Leclerc and Zelevinsky follows.

Corollary $28\left(\underline{(\underline{\mathbf{O P S}}))}\right.$ Every maximal weakly separated collection in $\left(\begin{array}{c}{[n]} \\ k\end{array}\right)$ has cardinality $k(n-k)+1$. Any such two maximal weakly separated collections are flip-connected.

One can observe that $\left(\begin{array}{c}{[n]} \\ k\end{array}\right)$ is a positroid corresponding to the top cell of the nonnegative part of the grassmannian. The corresponding strata has dimension $k(n-k)+1$. This number can also be read from the corresponding $\lrcorner$-diagram, which looks like a $k$ by $n-k$ rectangle with dots inside all the boxes.

Definition 29 Let $\mathcal{M}$ be a positroid having grassmann necklace $\mathcal{I}=\left(I_{1}, \cdots, I_{n}\right) . \mathcal{C} \subseteq \mathcal{M}$ is called a weakly separated collection inside $\mathcal{M}$ if it contains all $I_{i}$ for $i \in[n]$ and each pair of its elements are weakly separated. Maximal weakly separated collection is defined as maximal collections among weakly separated collections of $\mathcal{M}$.

Then we have the following generalization for general positroids:

Theorem $30((\overline{\mathbf{O P S}}))$ Fix any positroid $\mathcal{M} . \mathcal{C} \subseteq \mathcal{M}$ is a maximal weakly separated collection if and only if it is $\mathcal{F}_{\mathcal{G}}$ for some $\mathcal{G}$ a plabic graph of $\mathcal{M}$.

Corollary $31((\overline{\mathbf{O P S}}))$ Every maximal weakly separated collection of $\mathcal{M}$ has cardinality $\operatorname{dim}\left(S_{\mathcal{M}}\right)$. Any two maximal weakly separated collections of $\mathcal{M}$ are flip-connected. 


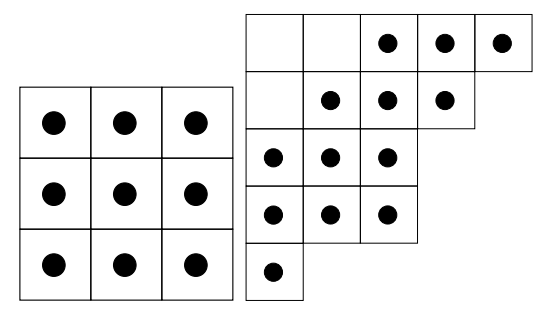

Fig. 9: Labeling the dots of a $\lrcorner$-diagram cooresponding to the top cell, a lattice path matroid.

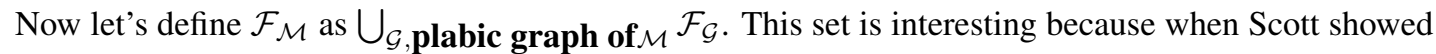
that the coordinate ring of the Grassmannian has a cluster algebra structure in $(\underline{S})$, one of the two main key steps was to show that $\mathcal{F}_{\left(\begin{array}{c}{[n]} \\ k\end{array}\right)}=\left(\begin{array}{c}{[n]} \\ k\end{array}\right)$. Now what follows from the above theorem is describtion of $\mathcal{F}_{\mathcal{M}}:$

Corollary $32 I \in F_{\mathcal{M}}$ if and only if $I$ is weakly separated with $I_{1}, \cdots, I_{n}$, where $\mathcal{I}=\left(I_{1}, \cdots, I_{n}\right)$ is the grassmann necklace of $\mathcal{M}$.

Now we will see a nice method to compute the cardinality of $F_{\mathcal{M}}$ using $\lrcorner$-diagrams.

Definition 33 Let us be given a $\lrcorner$-diagram. Choose $k$-columns and $k$-rows. Then we get $k^{2}$ positions, and if there are dots in every positions, we call that a $k$-by- $k$ full-minor. We say that every $\lrcorner$-diagram has exactly one 0-by-0 full-minor.

Theorem 34 Fix a positroid $\mathcal{M}$. Let $m_{i}$ denote the number of $i$-by-i full-minors inside the corresponding \lrcorner -diagram. Then $\left|F_{\mathcal{M}}\right|=\sum_{i=0}^{\infty} m_{i}$.

For each dot in the $\lrcorner$-diagram, if we label the dot with sum of number of all $i$-by- $i$ minors having that dot as the upper-left corner for all $i$, we get something more interesting. For top cells and lattice path matroids( When $\lrcorner$-diagram consists of a skew-young diagram with dots inside all the boxes.), we get the Pascal's triangle. This labeling gives us another way to compute $\left|F_{\mathcal{M}}\right|$.

Proposition $35\left|F_{\mathcal{M}}\right|$ can also be obtained by adding 1 to the sum of all the labels of the dots given as above.

Look at Figure 9 for an example. If we look at the left figure corresponding to the top cell $\left(\begin{array}{c}{[6]} \\ 3\end{array}\right)$, we can get $\left|F_{\left(\begin{array}{c}{[6]} \\ 3\end{array}\right)}\right|=1+5 * 1+2 * 1+3 * 2+6=20$.

It would be interesting to find a way to count the number of plabic graphs of a given cell. That is, a way to count the number of maximal weakly separated collection of a positroid.

Problem 36 What is the number of maximal weakly separated collection of a positroid $\mathcal{M}$ ?

\section{References}

[BGW] A. Borovik, I. Gelfand, N. White, Coxeter Matroids Birkhauser, Boston, 2003.

[BLSWZ] A. Björner, M. Las Vergnas, B. Sturmfels, N. White, and G. M. Ziegler. Oriented Matroids. Encyclopedia of Mathematics and Its Applications, vol. 46. Cambridge University Press, Cambridge, 1993. 
[G] D. Gale. Optimal assignments in an ordered set: an application of matroid theory, J. Combinatorial Theory 4(1968), 1073-1082.

[L] G. Lusztig. Introduction to total positivity, in Positivity in Lie theory: open problems, ed. J. Hilgert, J.D. Lawson, K.H. Neeb, E.B. Vinberg, de Gruyter Berlin, 1998, 133-145

[LZ] B. Leclerc, A. Zelevinsky. Quasicommuting families of quantum Plucker coordinates, American Mathematical Society Translations, Ser. 2 181, 1998.

[O1] S. Oh. Positroids and Schubert matroids, arXiv:0803.1018 [math.CO].

[O2] S. Oh. Contraction and restriction of positroids in terms of decorated permutations arXiv:0804.0882 [math.CO].

[OPS] S. Oh, A. Postnikov, D. Speyer. Weakly separated sets and plabic graphs, In preparation.

[P] A. Postnikov. Total positivity, Grassmannians, and networks, arXiv: math/ 0609764v1 [math.CO].

[S] J. Scott. Grassmannians and cluster algebras, Proc. London Math. Soc. (3) 92(2006), 345-380. 